\title{
Facile Synthesis of 5-Arylpent-4-enoates from the Baylis-Hillman Acetates
}

\author{
Yang Jin Im, Jeong Mi Kim, and Jae Nyoung Kim

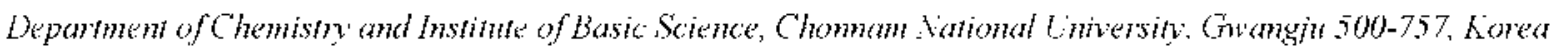 \\ Received June 7, 2002
}

Key Words : 5-Arylpent-4-enoates. Baylis-I lilman acetates, Decarbethosylation, DMN'

Recenty: Basavaial et al, have published some papers dealing with the Johnson-Claisen rearrangement of the Baylis-

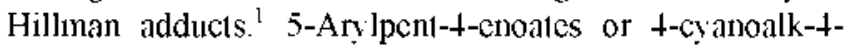
enoates can be obtained from the above reaction in moderate viclds. Shen et al. have also reported the synthesis of the latter compounds by using the sequential Michacl reaction and Horner-Wadworth-Emmons (HWE) reaction of phosplonates. 2

During our sludies on the Baylis-Hillman chemistry ${ }^{3}$ we found another efficient method for the synthesis of the abovementioned compounds. As shown in Scheme 1 the reaction of the Baylis-Hillman acclate 1a and dicthy] malonate (2a) in $\mathrm{CH}_{3} \mathrm{CN}$ in the presence of $\mathrm{K}_{2} \mathrm{CO}_{3}$ gave the allylic rearrangement product 3 a in good yield. The struclure of 3 a was exclusively $E$-form as in our previous papers. ${ }^{3}$ Trace amount (ca. 5\%) of the corresponding 7 -form was observed in 'H NMR specinum. The separation of $E$ and $7-$ form was difficult at this stage. Thus. we used the misiture directly in the next reaction wilhoul further purification, Following decarbethoxylation was conducted in $p$-xylene in the presence of 4-dimelly laminopyridine (4-DMAP). ${ }^{\text {tat }} 1$ th We could isolate the desired compound ta in $77 \%$ yicld. A1 1his stage. pure $4 \mathbf{a}-F$ could be separated casily from the minor component. 4a- $Z$. In the reaction. DABCO (1.4-diazabicyclo[2.2.2 loctanc) and DBN (1.5-diazabicyclo[4,3.0]non-

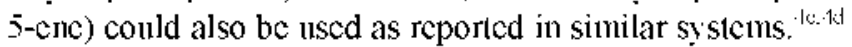
Howerer. the use of DMAP in refluxing xy lene gave the best results.

The representative results for the synthesis of the allylic rearrangement products 3a-g are summarized in Table 1. Besides of diethyl malonate (2a. entries 1-3) and dimetlỵ]
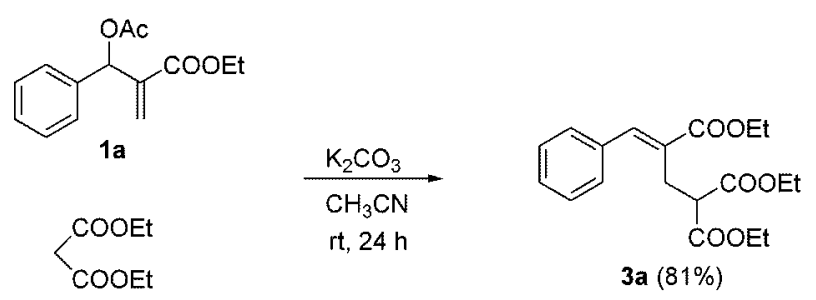

2a
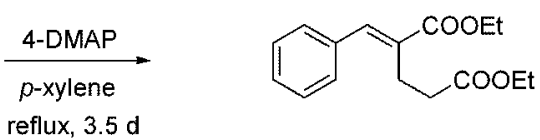

$4 a(77 \%)$
Table 1. Synthesis of allylic rearrangement products 3

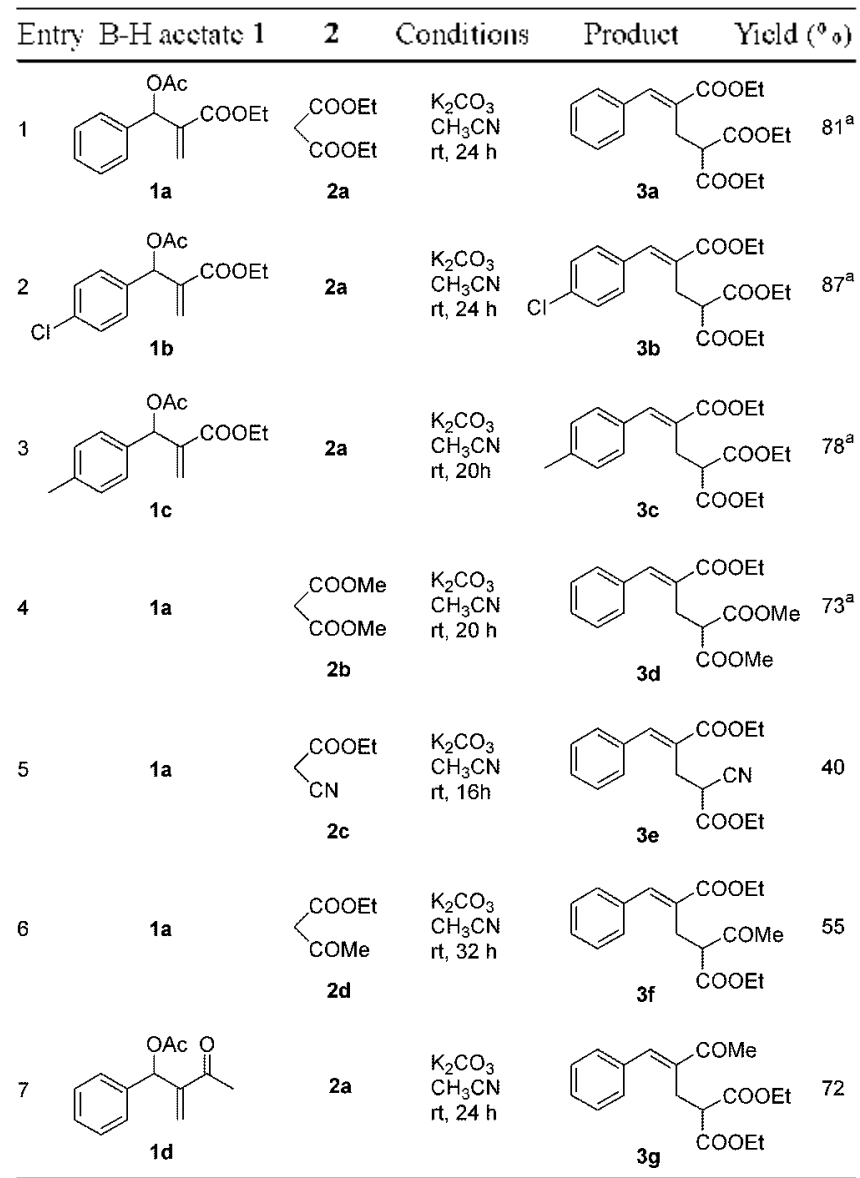

"Trace amounts (co. $5^{\circ}$ o) of the corresponding $Z$-isomer were obser'sed in lheir 'H NMR spectra.

malonate (2b. entry 4). some other activated methylenc compounds such as ctlyyl cyanoacclate (2c. cniry 5) and cllyyl acctoacctate (2d. cniry 6) gave similar results. The Baylis-Hillman acetate 1(l. derived from benzaldehyde and metlyyl vinyl ketone. gave 3g similarly (eniry 7). The results of sclective decarbethoxylation of 3a-g will 4-DMAP (5 cquiv) are summarized in Table 2.

A typical procedure for the synthesis of 3 a and $4 \mathbf{a}$ is as follows: To a stired solution of 1a (496 $\mathrm{mg} .2 .0 \mathrm{mmol})$ and dictlyy malonate $(360 \mathrm{mg} .2 .2 \mathrm{mmol})$ in $\mathrm{CH}_{i} \mathrm{CN}(5 \mathrm{~mL})$ was added $\mathrm{K}_{2} \mathrm{CO}_{\text {s }}(305 \mathrm{mg} .2 .2 \mathrm{mmol})$ and the mixture was stirred at room temperature for $2+\mathrm{l}$. After usual workup and column chromatographic purification (hexanc/cther. $8: 1$ )

Scheme 1 
Table 2. Synthesis of 5-arylpent-4-enoate derivatives 4

\begin{tabular}{|c|c|c|c|c|}
\hline Entry & 3 & Conditions & Products & Yicld $\left({ }^{\circ}{ }_{0}\right)^{\prime}$ \\
\hline 1 & 3a & $\begin{array}{l}\text { 4-DMAP ( } 5.0 \text { equiv.) } \\
\text { p-xylene } \\
\text { reflux, } 3.5 \text { days }\end{array}$ & $4 a$ & 77 \\
\hline 2 & $3 b$ & $\begin{array}{l}\text { 4-DMAP ( } 5.0 \text { equiv.) } \\
p \text {-xylene } \\
\text { reflux, } 3 \text { days }\end{array}$ & & 67 \\
\hline 3 & $3 c$ & $\begin{array}{l}\text { 4-DMAP ( } 5.0 \text { equiv) } \\
\rho \text {-xylene } \\
\text { reflux, } 6 \text { days }\end{array}$ & & 40 \\
\hline 4 & $3 d$ & $\begin{array}{l}\text { 4-DMA.P ( } 5.0 \text { equiv.) } \\
\text { p-xylene } \\
\text { reflux, } 2 \text { days }\end{array}$ & & 50 \\
\hline 5 & $3 e$ & $\begin{array}{l}\text { 4-DMAP ( } 5.0 \text { equiv) } \\
\text { p-xylene } \\
\text { reflux, } 2 \text { days }\end{array}$ & & 57 \\
\hline 6 & $3 f$ & $\begin{array}{l}\text { 4-DMAP ( } 5.0 \text { equiv.) } \\
\text { p-xylene } \\
120^{\circ} \mathrm{C}, 4 \text { days }\end{array}$ & 4 & 64 \\
\hline 7 & $3 \mathrm{~g}$ & $\begin{array}{l}\text { 4-DMAP (5.0 equiv.) } \\
\rho \text {-xylene } \\
\text { reflux, } 6 \text { days }\end{array}$ & 4 & 45 \\
\hline
\end{tabular}

"l'ure $E$-limm. "Products were obtained as clear wil except for $4 \mathbf{f}$ (mp th$\left.46{ }^{\prime \prime} \mathrm{C}\right)$.

3a was obtained as a clear oil. $565 \mathrm{mg}(81 \%){ }^{6}$ A stirred solution of 3a (3+8 $\mathrm{mg}$. $1.0 \mathrm{mmol}$ ) and +-DMAP $(610 \mathrm{mg} .5$ munol) in dry xylene $(3 \mathrm{~mL})$ was heated to reflux under nitrogen atmosphere for 3.5 days. After removal of the solvent and column chromatographic purification (hexane/ ether. $8:$ 1) ta was obtained as an oil. $213 \mathrm{mg}(77 \%) .{ }^{6}$

In conclusion we disclosed a facile synthetic method of sy nthetically useful 5-arylpent-t-enoate derivatives. ${ }^{1: 2}$

Acknow ledgment. This work was supported by the grant (R02-2000-00074) from the Basic Research program of the
Korea Science \& Engineering Foundation.

\section{References and Notes}

1. (a) Basavaiah. D.: P'andiaraju. S. Tetrahedron Lett. 1995. 36. 757 . (b) Basavaiah. D.: l’andiaraju. S.: Krishmamacharyulu. M. Symett 1996,747

2. (a) Shen, Y.: 7hang. 7. Symh. Commm. 2000, 30. 445. (b) Shen. Y.: 7.hang. 7. J. Chem. Ris. (S) 1949. 556.

3. (a) Kim. J. N.: Lee. H. J.: Lee. K. Y.: Gone. I. H. Synlett 2002. 173. (b) Geng. J. H.: Im. Y. J.: Lee. K. Y.: Kim. J. N. Tetrahedron lett. 2002. 43. 1247. (c) Chung. Y. M.: Gong. I. H.: Kim. I: H.: Kim, I. N. Tetrahedrm Leff. 2001, 12,9023. (d) Kim. J. N.: Kim. II. S.: Gong. I. II.: Chung. Y. M. Tetrathe dirm Letf 2001. 42.8341. (c) Kim. J. N.: Im, Y. J.: Gong. J. I I.: I.ee. K. Y. Fetrohedron Lett. 2001. 42. 4195. (D) Kim. I. N.: Lee. H. J.: Lee. K. Y.: Kim. H. S. Tetrahedron Lett. 2001. 42. 3737. (g) Kim. H. S.: Kim. 'I. Y.: Lee. K. Y.: Chung. Y. M.: Lee. H. I.: Kim. I. N. Tetrohedron Lett. 2000. 41, 2613. (h) Kim. I. N.: I.ee. K. Y: Kim. II. S.: Kim. T. Y. Ory. Leff. 2000, 2. 34.3. (i) I.ce, HI. J.: Scong, M. R.: Kim. J. N. Tetrathedron Lett. 1998. 39. 6223. (i) I. $\alpha$. II. J.: Kim. II. S.: Kim. J. N. Tetrahedron lett 1999. 40.4363 , (k) Chung. Y. M.: Lee. H. J.: Hwang. S. S.: Kim. J. N. Bull. Korean (hem. Soc, 2001. 22. 799. (1) Im. Y. J.: Kim. J. M.: Mun. J. H.: Kim. J. N. Bull. Kortan Chem. Soc. 2001, 22. 349. (m) Kim, I. N.: I ce. K. Y. Cumb Organic Chemistry 2002.6,627.

4. (a) Taber, D. F.: Anedio. I. C.. Jr.; Gulito. F. J. Ory. Chem 1989. 54. 3474. (b) Tuber. D. F.: Amedio. J. C.. Jr.: Palel. Y. K. J. Org. (Wem. 1985. 50. 3618. (c) Miles. D. H.: Huane. B.-S. J. Org. Chem. 1976. H. 208. (d) Huang. B.-S.: Parish. E. J.: Miles. D. H. J. Org. Chem. 1974. 39.2647.

5. Decarbethoxylation of 3a with $\mathrm{DABCO}$ (10 equiv) in refluxing xylene gave $4 a$ in $45^{\circ}$ o vield after 2 days. The use of DBN (5 equiv) in similar reaction conditions gave intractable mixtures.

6. Selecled dala for 3a and 4a. 3a: oil: IR (KBr) 1746. 1733. 1707 $\mathrm{cm}^{-1}$ : 'H NMR (CDCl $) \delta 1.15(\mathrm{l} . J-7.2 \mathrm{H} .6 \mathrm{H}) .1 .34(\mathrm{I} . j-7.2$

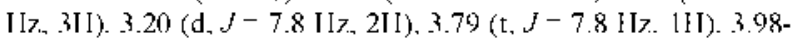
$4.15(\mathrm{~m}, 4 \mathrm{HI}) .4 .27(\mathrm{q} . j-7.2 \mathrm{Il}, 2 \mathrm{II}) .7 .25-7.38(\mathrm{~m} .5 \mathrm{H}) .7 .77(\mathrm{~s}$ III): ${ }^{13} \mathrm{C}$ NMR $\left(\mathrm{CDCl}_{3}\right) \delta$ 13.79. 14.13. 26.15. 50.42. 60.87. 61.18. 127.83. 128.14. 128.46. 129.01. 134.92. 141.44. 167.32. 168.72. 4a: oil: IR (KBr) 1734. $1707 \mathrm{~cm}^{-1}$ : ${ }^{1} \mathrm{H} \mathrm{NMR}\left(\mathrm{CDCl}_{3}\right) \delta$ $1.15(\mathrm{t} . J-7.2 \mathrm{H} \measuredangle .3 \mathrm{H}) .1 .27(\mathrm{t} . J-7.2 \mathrm{H} \measuredangle .3 \mathrm{H}) .2 .45-2.5 \mathrm{l}(\mathrm{m}$. 2II). $2.77-2.8 .3(\mathrm{~m}, 2 \mathrm{H}) \cdot 4.0 .3(\mathrm{c} . J-7.2 \mathrm{HJ} .2 \mathrm{H}) .4 .20(\mathrm{q} . J-7.2$ II7, 2HI). 7.27-7..32 (m, 5II), 7.65 (s, III): $\left.{ }^{13} \mathrm{C} \mathrm{NMR} \mathrm{(CDCl}\right) \delta$ 14.07. 14.19. 22.99. 33.44,60.32,60.81. 128.47. 128.49. 129.05 . 131.38. 135.21. 140.01. 167.69. 172.61. 